INPLASY

PROTOCOL

To cite: Gupta et al.

Sanitization of footwear and textiles for eradication of causal agents of superficial fungal infections: protocol for a systematic review. Inplasy protocol 2021110070. doi: 10.37766/inplasy2021.11.0070

Received: 17 November 2021

Published: 17 November 2021

Corresponding author: Aditya Gupta

agupta@mediproberesearch.com

Author Affiliation:

Mediprobe Research Inc.

Support: Self-financed.

Review Stage at time of this submission: Preliminary searches.

Conflicts of interest: None declared.

\section{Sanitization of footwear and textiles for eradication of causal agents of superficial fungal infections: protocol for a systematic review}

Gupta, AK'; Simkovich, AJ2; Hall, DC³.

Review question / Objective: To compile evidence and determine the utility of various methods used to sanitize footwear and textiles, and the use of novel antimicrobial materials for eradication of pathogens known to cause superficial fungal infections of the foot.

Rationale: Fungal infections of the feet such as onychomycosis are common, affecting approximately $6 \%$ of the global population. There are a number of available treatment methods for onychomycosis, with topical (e.g., efinaconazole, tavaborole, ciclopirox), oral (e.g., terbinafine, itraconazole, fluconazole), or a combination of both, being the most popular. Sanitization of shoes, socks/stockings, and other textiles (as well as the feet themselves through proper hygiene) is integral to the reduction, spread, and recurrence of superficial fungal infection. The goal of the present review is to examine the currently available methods of sanitization for footwear and textiles against superficial fungal infections, and assessing which are effective or not.

INPLASY registration number: This protocol was registered with the International Platform of Registered Systematic Review and Meta-Analysis Protocols (INPLASY) on 17 November 2021 and was last updated on 17 November 2021 (registration number INPLASY2021110070).

\section{INTRODUCTION}

Review question / Objective: To compile evidence and determine the utility of various methods used to sanitize footwear and textiles, and the use of novel antimicrobial materials for eradication of pathogens known to cause superficial fungal infections of the foot.

Rationale: Fungal infections of the feet such as onychomycosis are common, affecting approximately $6 \%$ of the global population. There are a number of available 
treatment methods for onychomycosis, with topical (e.g., efinaconazole, tavaborole, ciclopirox), oral (e.g., terbinafine, itraconazole, fluconazole), or a combination of both, being the most popular. Sanitization of shoes, socks/ stockings, and other textiles (as well as the feet themselves through proper hygiene) is integral to the reduction, spread, and recurrence of superficial fungal infection. The goal of the present review is to examine the currently available methods of sanitization for footwear and textiles against superficial fungal infections, and assessing which are effective or not.

Condition being studied: Toenail onychomycosis, also known as tinea unguium.

\section{METHODS}

Search strategy: Both Pubmed and Scopus databases will be searched using the following search strings: ((onychomycosis OR superficial mycoses OR fungal infection OR tinea unguium) AND (sanitiz* OR hygien* OR wash* OR clean* OR laund*)) AND (sock* OR shoe* OR textile* OR stocking*) And ((onychomycosis OR superficial mycoses OR fungal infection OR tinea unguium) AND (sanitiz* OR hygien* OR wash* OR clean* OR laund* OR disinf*)) AND (sock* OR shoe* OR textile* OR stocking*).

Participant or population: Studies including human patients and in vitro/ex vivo studies will be included in this review, therefore populations will include: subjects diagnosed with onychomycosis; participants of all ages and races/ ethnicities. Additionally, footwear and textiles subjected to inoculation with cultured fungi will be included.

Intervention: Various methods used to sanitize footwear and textiles: sanitization powder and chemicals, laundering, ultraviolet irradiation, ozone treatment, non-thermal plasma, microwave radiation, treatment with essential oils, treatment with natural plant extracts. The use of antifungal and antimicrobial materials in the construction of footwear and textiles will also be included as a potential intervention.

Comparator: Comparators can include: (1)no treatment (i.e., nothing)(2)placebo/ mock/sham treatment.

Study designs to be included: Evidence will be gathered from primary research articles including case reports, clinical trials, in vitro and ex vivo studies.

Eligibility criteria: Studies that investigated the utility of sanitization of footwear or other textiles, or the utility of novel antimicrobial materials for the eradication and recurrence prevention of causal agents of superficial mycoses. Only articles published in English will be included; there will be no date restrictions.

Information sources: Searches will be conducted in the PubMed and Scopus search engines.

Main outcome(s): Main outcomes will include the inhibition of fungal growth and the eradication of fungal organisms.

Additional outcome(s): None.

Data management: Data will be organized into spreadsheets.

Quality assessment / Risk of bias analysis: The quality of evidence across studies will be evaluated using the Grading of Recommendations, Assessment, Development and Evaluations (GRADE) framework

Strategy of data synthesis: The goal of this project is to conduct a systematic review

Subgroup analysis: None.

Sensitivity analysis: None.

Language: English.

Country(ies) involved: Canada.

Keywords: Onychomycosis; superficial mycoses; Tinea unguium; sanitization. 
Contributions of each author:

Author 1 - Aditya Gupta - Proposed the paper, will oversee the search process, make final decisions when other authors have conflict for article inclusion (if any), edit and review manuscript throughout its completion.

Email: agupta@mediproberesearch.com

Author 2 - Aaron Simkovich - Partake in initial searching, make decisions of exclusion of articles based on title, abstract, or contents, assist in writing and editing manuscript.

Email: asimkovich@mediproberesearch.com Author 3 - Deanna Hall - Partake in initial searching, make decisions of exclusion of articles based on title, abstract, or contents, author of bulk of the manuscript, assist in editing.

Email: dhall@mediproberesearch.com 